\title{
Editorial
}

\section{Enabling Technologies for 5G Mobile Systems}

\author{
Qilian Liang, ${ }^{1}$ Tariq S. Durrani, ${ }^{2}$ Jing Liang, ${ }^{3}$ and Xin Wang ${ }^{4}$ \\ ${ }^{1}$ Department of Electrical Engineering, University of Texas at Arlington, Arlington, TX 76019-0016, USA \\ ${ }^{2}$ Department of Electronic and Electrical Engineering, University of Strathclyde, Glasgow G1 1XW, UK \\ ${ }^{3}$ College of Electronic Engineering, University of Electronic Science and Technology, Chengdu 611731, China \\ ${ }^{4}$ Qualcomm Inc, 1650 Technology Drive, San Jose, CA 95110, USA
}

Correspondence should be addressed to Qilian Liang; liang@uta.edu

Received 22 February 2016; Accepted 22 February 2016

Copyright (C) 2016 Qilian Liang et al. This is an open access article distributed under the Creative Commons Attribution License, which permits unrestricted use, distribution, and reproduction in any medium, provided the original work is properly cited.

Mobile information systems have been facing the challenges of the continuously increasing demand for the high data rates and mobility required by new wireless applications. 4G/LTE wireless networks now enable high-speed mobile web videos, IP telephony, video gaming, mobile high-definition (HD) TV, video conferencing, and even mobile 3D TV. Because of the convenience and freedom afforded by high-performance video-optimized mobile devices, wireless video traffic is exploding. Indeed, the wireless telecommunication industry is facing a watershed "moment" where foreseeable capacity may soon fail to meet the demand. The current trend of annual doubling of wireless data traffic is expected to continue. Given the accelerating sales of tablet computers, which can consume several times as much spectrum as smart phones, these trends are likely to continue. Research on 5G wireless communications has started and developed rapidly. It is expected that $5 \mathrm{G}$ wireless infrastructure will be deployed beyond 2020. However, enabling technologies for $5 \mathrm{G}$ mobile information systems such as massive MIMO, new modulation, and waveform design are still in their infancy. More efforts from this community are truly needed to make $5 \mathrm{G}$ mobile systems reach their full fruition.

This special issue contains 11 papers selected from submissions to the open call for papers. These papers highlight some of the current research interests and achievements in the area of enabling technologies for $5 \mathrm{G}$ mobile systems.

Interference is an important and challenging problem faced by the D2D underlaid cellular networks. The paper, "Exploiting the User-Level Interference Based on Network Coding in D2D Underlaid Cellular Networks," by Y. Guo et al., focused on the user-level interference under multicasting scenarios.

Delay and throughput are important metrics for network performance. The paper, "End-to-End Delay Analysis in Cognitive Radio Ad Hoc Networks with Different Traffic Models," by J. Gao et al., analyzes the end-to-end delay of cognitive radio ad hoc networks for two traffic models: backlogged and geometric.

Interference alignment (IA) is an effective technique to eliminate the interference among wireless nodes. In a multiinput multioutput (MIMO) cognitive radio system, multiple secondary users can coexist with the primary user without generating any interference by using the IA technology. However, few works have considered the fairness of secondary users. In the paper, "Interference Alignment and Fairness Algorithms for MIMO Cognitive Radio Systems," by F. Zhao et al., not only the interference is eliminated by IA, but also the fairness of secondary users is considered by two kinds of algorithms.

Massive multiple input, multiple output (M-MIMO) technologies have been proposed to scale up data rates reaching gigabits per second in the forthcoming $5 \mathrm{G}$ mobile communications systems. However, one of the crucial constraints is dimension in space to implement the M-MIMO. The paper, "Diversity and Multiplexing Technologies by 3D Beams in Polarized Massive MIMO Systems," by X. Su and K. Chang, presents a polarized M-MIMO (PMMIMO) system associated with $3 \mathrm{D}-\mathrm{BF}$ applications, where the system architectures for diversity and multiplexing technologies achieved by polarized $3 \mathrm{D}$ beams are provided. 
How to make more small cells in sleeping state for energy saving in ultradense small cell system has become a research hotspot. The paper, "The Resource Mapping Algorithm of Wireless Virtualized Network for Saving Energy in Ultradense Small Cells," by S. Zou et al., proposes a new wireless resource mapping algorithm for saving energy in ultradense small cells which has been put forward when wireless resource amount is satisfied in every small cell.

The key of wireless power transfer technology rests on finding the most suitable means to improve the efficiency of the system. The wireless power transfer system applied in implantable medical devices can reduce the patients' physical and economic burden because it will achieve charging in vitro. In the paper, "Optimal Design and Analysis of the Stepped Core for Wireless Power Transfer Systems," by X. Zhang and X. Zhang, the transmitter coil is designed and optimized.

The paper, "Game-Theoretic Beamforming and Power Allocation in MIMO Cognitive Radio Systems with Transmitter Antenna Correlation," by F. Zhao et al., considers antenna correlation at the transmitter in practical cognitive MIMO systems. What is more, a game-theoretic framework is conducted to analyze the optimum beamforming and power allocation such that each user maximizes its own rate selfishly under the transmit power constraint and the primary user (PU) interference constraint. The design of the cognitive MIMO system is formulated as a noncooperative game, where the secondary users (SUs) compete with each other over the resources made available by the PUs.

Compressive sensing theory can be applied to reconstruct the signal with far fewer measurements than what is usually considered necessary. While, in many scenarios, such as spectrum detection and modulation recognition, it is only expected to acquire useful characteristics rather than the original signals, where selecting the feature with sparsity becomes the main challenge. With the aim of digital modulation recognition, the paper, "Feature-Based Digital Modulation Recognition Using Compressive Sampling," by Z. Sun et al., mainly constructs two features which can be recovered directly from compressive samples.

A device-to-device (D2D) group works as relay nodes to aid in information delivery from a source to a destination in cellular communication network. The paper, "Incorporating D2D to Current Cellular Communication System," by M. Dai et al., proposes a communication mechanism to aid in traditional cellular communication and correspondingly borrow some channel resource from traditional cellular communication system for D2D communication.

With the widespread use of Internet, the scale of mobile data traffic grows explosively, which makes $5 \mathrm{G}$ networks in cellular networks become a growing concern. Recently, the ideas related future network, for example, Software Defined Networking (SDN), Content-Centric Networking (CCN), Big Data, have drawn more and more attention. The paper, "An Optimal Routing Algorithm in Service Customized 5G Networks," by H. Yao et al., proposes a service-customized 5G network architecture by introducing the ideas of separation between control plane and data plane, in-network caching,
Big Data processing and analysis to resolve the problems traditional cellular radio networks face.

In 5G mobile network, millimeter wave (mmWave) and heterogeneous networks (Hetnets) are significant techniques to sustain coverage and spectral efficiency. The paper, "SDN Controlled mmWave Massive MIMO Hybrid Precoding for 5G Heterogeneous Mobile Systems," by S. Sun et al., proposes the utilization of the hybrid precoding to overcome hardware constraints on the analog-only beamforming in mmWave systems.

\section{Acknowledgments}

We would like to thank all authors for contributing papers to this special issue. We appreciate the staff of this journal for their support in editing this special issue.

Qilian Liang

Tariq S. Durrani Jing Liang Xin Wang 

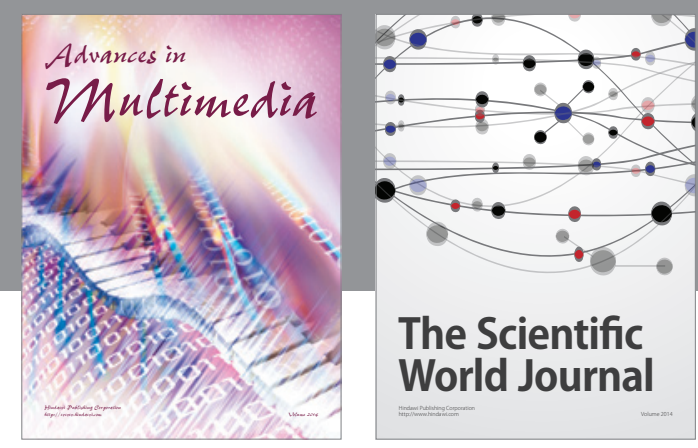

The Scientific World Journal
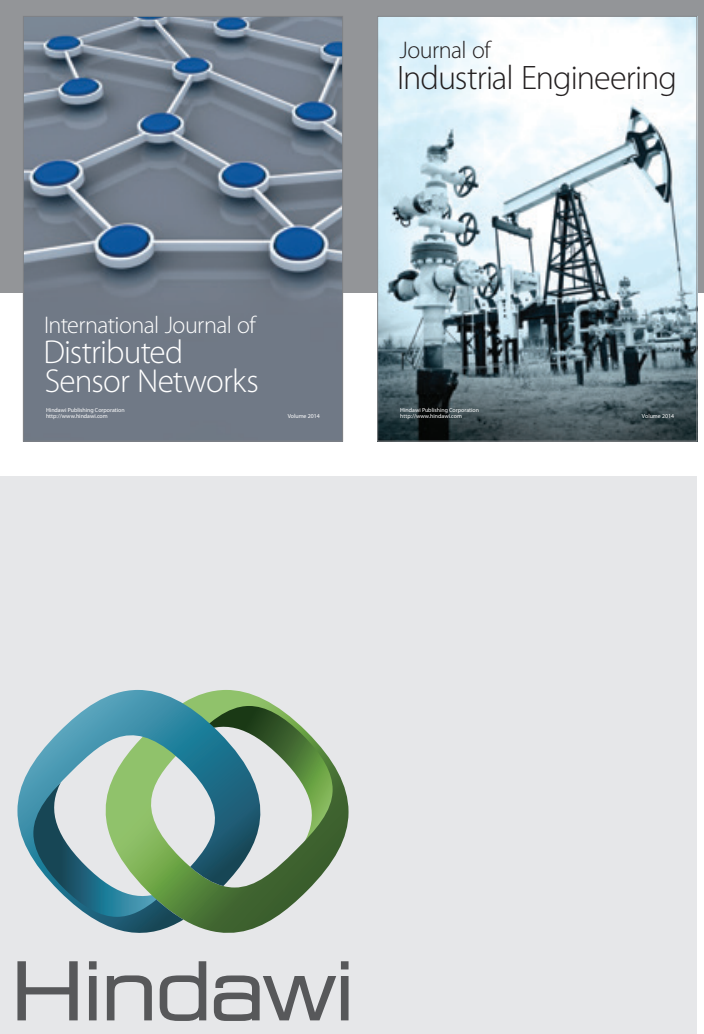

Submit your manuscripts at

http://www.hindawi.com

\section{Computer Networks} and Communications
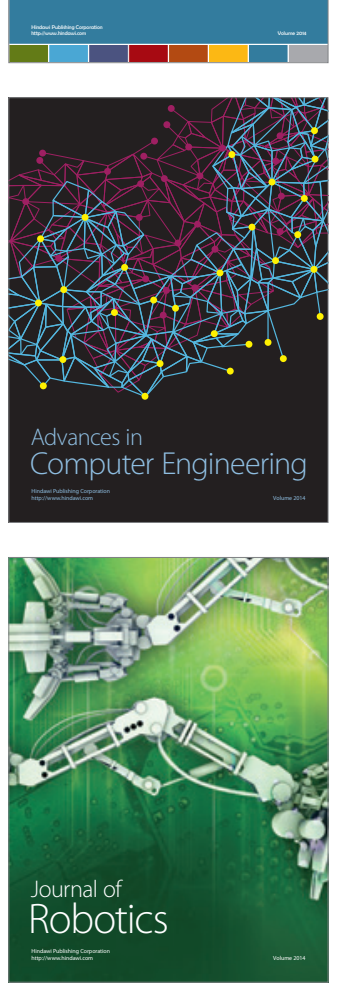
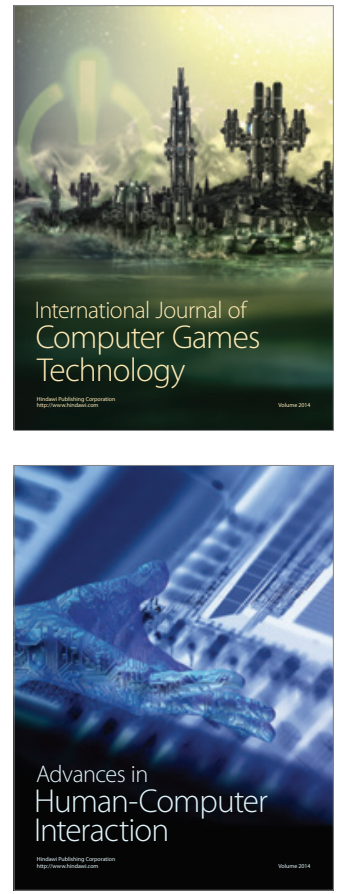
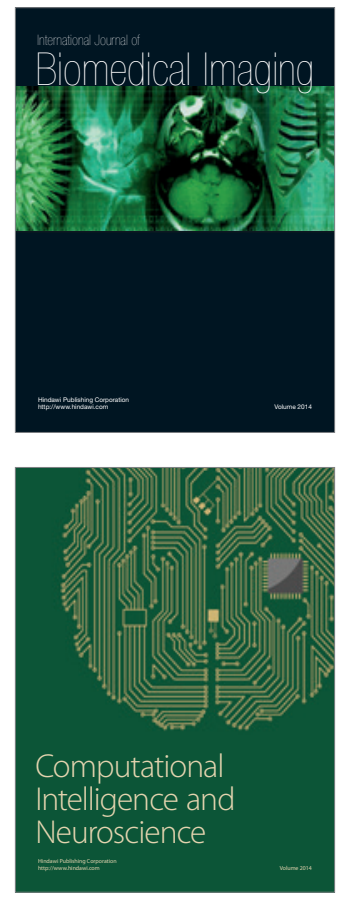
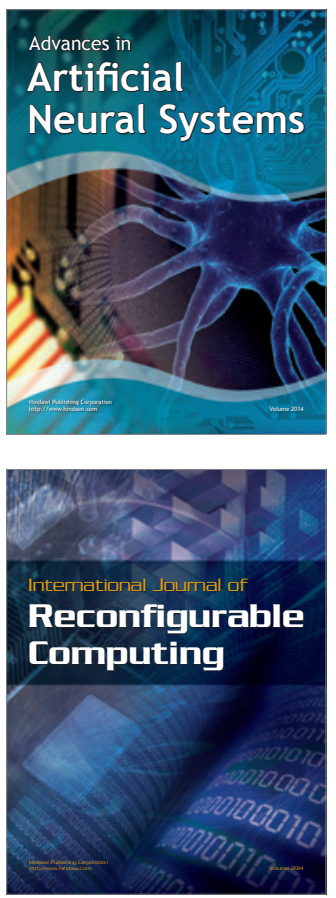
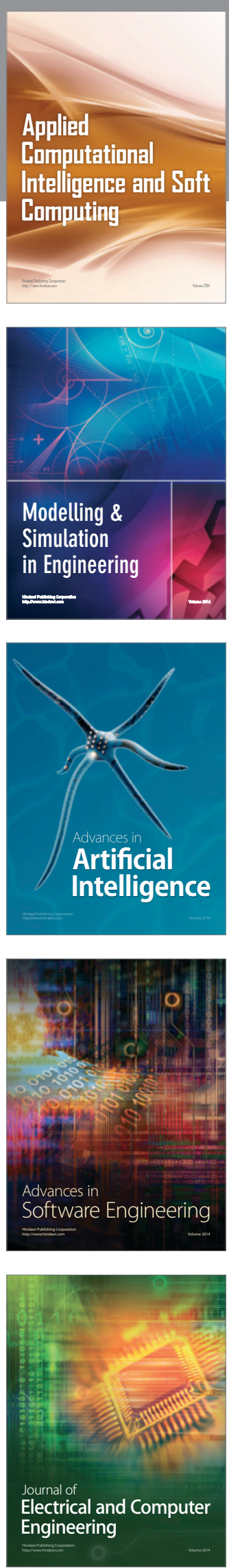